\title{
MicroRNA (miRNA) Regulation in Glioma: Implications in Development, Progression, Grading, Prognosis and Therapy
}

\author{
Kumaravel Somasundaram*, Soumya Alige Mahabala Rao \\ and Zahid Nawaz \\ Department of Microbiology and Cell Biology, Indian Institute of Science, Bangalore
}

India

\section{Introduction}

Neoplasms, which arise in cells originating from central nervous system (CNS) or peripheral nervous system (PNS) result in a class of tumours called neuroectodermal tumours. Generally after development ceases, neurons become post-mitotic and only the glial cells retain the capacity to divide and proliferate. So it is quite appreciable that most of the brain tumours are of glial origin and are referred as gliomas. Based on the cell type involved in malignancy, gliomas can be astrocytomas (astrocytes), oligodendrogliomas (oligodendrocytes), oligoastrocytomas (mixture of astrocytes and oligodendrocytes) and ependymomas (ependymal cells). Astrocytomas account for more than $60 \%$ of all primary brain tumours hence forming the majority of neoplasms of the CNS. On the other hand, neurofibroma and schwannoma are the two most common glial tumours of the PNS.

According to WHO (2007) classification, astrocytomas are classified into grades I-IV based on the intensity of malignancy as determined by histopathological criteria. Grade I (Pilocytic astrocytoma; PA) gliomas are generally benign and often circumscribed, whereas grade II (Diffuse astrocytoma; DA), III (Anaplastic astrocytoma; AA) and IV (Glioblastoma; GBM) gliomas are malignant and they diffusely infiltrate the brain. The most malignant and common form of infiltrating astrocytic tumours is glioblastoma with a median survival of less than one year. GBM can be further divided into two subtypes based on certain genetic and clinical features: primary and secondary GBM. Primary GBM arises as a de novo process, without any antecedent history of low grade lesions, whereas secondary GBM develops progressively from lower grades, generally over a period of 5-10 years.

Like any other neoplasm, the underlying cause of gliomagenesis is the genomic alterations like deletions, amplifications, mutations, aberrant DNA methylation and chromosomal rearrangements, which result in the activation of oncogenes and inactivation of tumour suppressors. With the discovery of hundreds of miRNA genes in the past few years that produce non-coding RNA transcripts with no significant open reading frame, it has become evident that the genomic complexity of the cancer cell is far greater than expected.

miRNAs are single-stranded non coding RNAs of 19-25 nucleotides in length, generated from endogenous transcripts that contain a local hairpin structure. They function as guide

${ }^{*}$ Corresponding author 
molecules in post-transcriptional gene silencing by base pairing with target mRNAs, leading either to the cleavage of the target mRNA or its translational repression. miRNAs are one of the largest gene families, accounting for $\sim 1 \%$ of the genome (Kim, 2005). According to recent studies, miRNAs play key roles in diverse regulatory pathways which control development, differentiation, apoptosis, cell proliferation and organogenesis. miRNAs and their targets seem to form intricate regulatory networks, as a single miRNA can bind to and regulate different mRNA targets and conversely, a single mRNA can be synergistically controlled by multiple miRNAs. Recently, the work by the Bartel and Burge laboratories predicted that over one third of the human genes are regulated by miRNAs (Lewis et al., 2005).

The location of miRNA genes in fragile sites of the genome provides a circumstantial evidence of an etiological role of miRNAs in tumour formation. Because miRNAs are generally negative regulators of gene expression, changes in the amounts of these RNAs can be tumorigenic if they target a tumour suppressor or an oncogene. For example, over accumulation of an miRNA that targets a tumour suppressor would result in less of that protective factor. In contrast, reduced accumulation of an miRNA that targets a protooncogene could lead to excessive amounts of the oncogenic protein. Either way, the net outcome is an imbalance in the activities of tumour suppressor genes and oncogenes, hence resulting in malignancy.

We review here the discovery and biology of miRNAs including their structure, regulation and biogenesis along with different methods available today to assay miRNA expression. A detailed account of the literature on differential miRNA expression and their functional characterization in glioma is provided. In addition, the potential utility of miRNAs in glioma diagnosis, classification, prognosis and targeted therapy is also discussed.

\section{Discovery and biology of miRNAs}

miRNAs were initially discovered as small temporal RNAs (stRNAs) that played a role in the developmental transitions in Caenorhabditis elegans. The first miRNA to be discovered was lin-4 in C. elegans in the year 1993 (Lee et al., 1993). The discovery of lin-4 and its target specific translational repression pointed to a new way of gene regulation. In the year 2000, the second miRNA, let-7 was discovered in the same organism and since then a huge number of miRNAs have been discovered. A total of 15172 miRNA entries have been recorded so far from various organisms (Release 16, miRBase) which also include 1048 miRNAs from Homo sapiens (http:// www.mirbase.org/).

A small RNA must fulfill the following criteria to be classified as an miRNA. Its expression should be authenticated by hybridization to a size-fractionated RNA sample by employing northern blotting. It should be detected by methods like PCR after reverse transcription of RNA (RT-PCR), primer extension analysis, RNase protection assay and microarray. However, northern blotting remains the method of choice for the confirmation of miRNAs, as the blot generally detects both the mature form (a 22-nucleotide band) and the hairpin precursor (a $\sim 70$-nucleotide band) of the predicted miRNA. The small RNA sequence must exist in the stem portion of the hairpin precursor. The precursors are $\sim 60-80$ nucleotides in animals, but the lengths are more variable in plants. In addition, the small RNA sequences should be conserved phylogenetically. Lastly, the evidence can be consolidated if the precursor of the predicted miRNA accumulates in ablated Dicer background. However, this criterion is not in vogue, because of the technical challenges that are involved in depleting Dicer in different cells. 


\section{1 miRNA gene structure}

Most of the miRNA genes are transcribed as independent transcription units. The transcripts thus synthesized are referred as primary miRNAs and are equipped with a 7methyl guanosine cap in the $5^{\prime}$ end as well as a poly A tail in the $3^{\prime}$ end which are the unique features of class II gene transcripts. This evidence supports the fact that majority of miRNAs, if not all, are transcribed by RNA polymerase II (Lee et al., 2004). Treatment of human cells with a-amanitin at levels which inhibit RNA polymerase II activity, also brings down the global levels of primary miRNAs. Furthermore, chromatin immunoprecipitation (ChIP) experiments have also revealed the physical association of RNA polymerase II with miRNA promoters and thereby establishing the fact that miRNAs are RNA polymerase II transcribed.

miRNAs can be transcribed either individually i.e. a single primary transcript gives rise to a single mature miRNA or they can be transcribed in a polycistronic fashion, in which case distinct miRNAs are produced from a single primary transcript. The miRNAs arising from a single gene cluster can be related to each other, suggesting that the miRNAs might have assembled as a result of gene duplication and may be playing a synergistic role in the production of a particular phenotype.

Based on the genomic location of the transcription units of miRNAs, they can be grouped broadly as: intergenic miRNAs and intragenic miRNAs. Intergenic miRNAs exist as independent genetic elements, their transcription being driven by their own promoter. The intragenic class can be either intronic or exonic in nature owing to the fact whether the stem loop sequence of the precursor miRNA comes from the intron or exon of the protein coding gene or sometimes a non-protein coding gene. In both types, the miRNA gene can be mono or polycistronic. The expression of intragenic miRNAs can be either controlled by their own independent promoter or the miRNAs would be co-transcribed with the parent gene, in which case the pre-miRNA would be processed subsequent to intron splicing. Some miRNAs derive their stem loop sequence from rather short introns and hence bypass the processing action of Drosha and are called Mirtrons (Ruby et al., 2007).

\section{2 miRNA gene regulation}

The expression of miRNAs can be modulated at a number of levels, which broadly include the transcription of the primary transcript and the multiple steps of the miRNA biogenesis. But it continues to remain a dilemma that which step is regulated and how the regulation is achieved. Regulation at the level of transcription seems more likely to be the major control mechanism. A number of RNA polymearse II associated transcription factors regulate the expression of miRNA genes, for instance TP53, a tumour suppressor, transactivates the miR34 family of miRNAs (He et al., 2007). Overall, the expression of a particular miRNA seems to be under the control of developmental and tissue specific signalling. Apart from this, there are reports that miRNAs are also modulated post transcriptionally. Post transcriptional regulation is attributed to differential Drosha processing. An example of post transcriptional regulation involves the induction of miR-21 in response to bone morphogenetic protein (BMP) / transforming growth factor beta (TGF- $\beta$ ) signalling without actual transcriptional activation (Davis et al., 2008). TGF- $\beta$ and BMP signalling activates the Smad mediated Drosha processing of the pri-miR-21.

Majority of miRNA genes being transcribed by the same RNA polymerase as protein coding genes, the mechanisms of epigenetic control known for protein coding genes are likely to be applicable to miRNA loci as well. Aberrant DNA methylation of tumour suppressor genes is 
a common occurrence in cancer; accordingly several miRNA loci including miR-9-1, miR193a, miR-137, miR-342, miR-203 and miR-34b/c, are found to be hypermethylated in multiple human cancers (Lujambio et al., 2008). Conversely, the let-7a-3 locus is hypomethylated in lung adenocarcinoma and elevated expression of this locus results in enhanced oncogenic gene transcription (Brueckner et al., 2007;). miRNA promoters are also regulated by histone modifications during development and pathogenesis (Davis and Hata, 2009).

\subsection{Biogenesis of miRNAs}

miRNA biogenesis involves initial transcription as a largely unstructured precursor, termed as primary miRNA (pri-miRNA), which is subsequently processed in the nucleus to give rise to a 70-nucleotide precursor miRNA hairpin intermediate (pre-miRNA). The premiRNA gets transported to the cytoplasm where it undergoes further processing to give rise to a mature miRNA (Figure.1).

Most of the miRNA genes are transcribed by RNA polymerase II to generate a stem-loop containing primary miRNA (pri-miRNA), which can range in size from hundreds of nucleotides to tens of kilobases (Lee et al., 2004). Like mRNAs, RNA polymerase II transcribed pri-miRNAs contain 5' cap structures, are polyadenylated, and may be spliced (Cai et al., 2004). The pri-miRNA is processed within the nucleus by a multiprotein complex called the microprocessor. The microprocessor is a $650 \mathrm{kDa}$ complex in humans and is constituted by two key components: Drosha, an RNase III enzyme and DiGeorge syndrome critical region 8 gene (DGCR8), a double-stranded RNA-binding domain (dsRBD) protein (Gregory et al., 2004; Landthaler et al., 2004). DGCR8 is also known as Pasha in D. melanogaster and C. elegans. The activity of Drosha and DGCR8 results in the production of a $\sim 70$ nucleotide hairpin structure known as the precursor miRNA (pre-miRNA). The 2nucleotide 3' overhang, characteristic of RNase III mediated cleavage, is recognized by exportin-5, which transports the pre-miRNA into the cytoplasm via a Ran-GTP-dependent mechanism (Bohnsack et al., 2004; Lund et al., 2004; Yi et al., 2003). The pre-miRNA is finally cleaved off the loop to produce the mature miRNA duplex about 22 nucleotide in length by Dicer, yet another member of RNase III superfamily of bidentate nucleases.

Dicer is accompanied by dsRBD proteins like TRBP (TAR-RNA binding protein) (Chendrimada et al., 2005; Lee et al., 2006). Dicer and TRBP recruit members of the Argonaute family and the trimeric complex thus formed is referred to as RNA Induced Silencing Complex (RISC) (Gregory et al., 2005; Maniataki and Mourelatos, 2005). Argonaute proteins form the catalytic component of the RISC complex as they aid in strand selection of the miRNA duplex in addition to the possession of slicer activity by virtue of which they slice the target mRNA. In mammals, Ago2 is the sole enzyme equipped with endonucleolytic activity (Liu et al., 2004; Meister et al., 2004). One of the miRNA strands which has relatively less stability at the $5^{\prime}$ end is incorporated into the RISC complex while as the other miRNA strand is generally degraded (Du and Zamore, 2005). It is often possible that both strands of the miRNA duplex could function as mature miRNAs but their abundance may be drastically different (Ruby et al., 2006).

The miRNA then guides the RISC to specific mRNA targets, the basis of which is sequence complementarity generally in the 3' UTR of the target mRNA. In cases of perfect or near perfect complementarity to the mRNA, the target mRNA can be cleaved and degraded and if there is less complementarity, it results in translational repression (Hutvagner and 
Zamore, 2002). Most animal miRNAs bind imperfectly to their targets and promote translational repression, rather than cleavage and degradation. However, the seed sequence representing 2-8 nucleotides from the $5^{\prime}$ end of miRNA requires a perfect complementarity. The mechanism of translational repression is somewhat vague with some evidences suggesting that miRNAs block translational initiation while as others suggest a block in elongation.

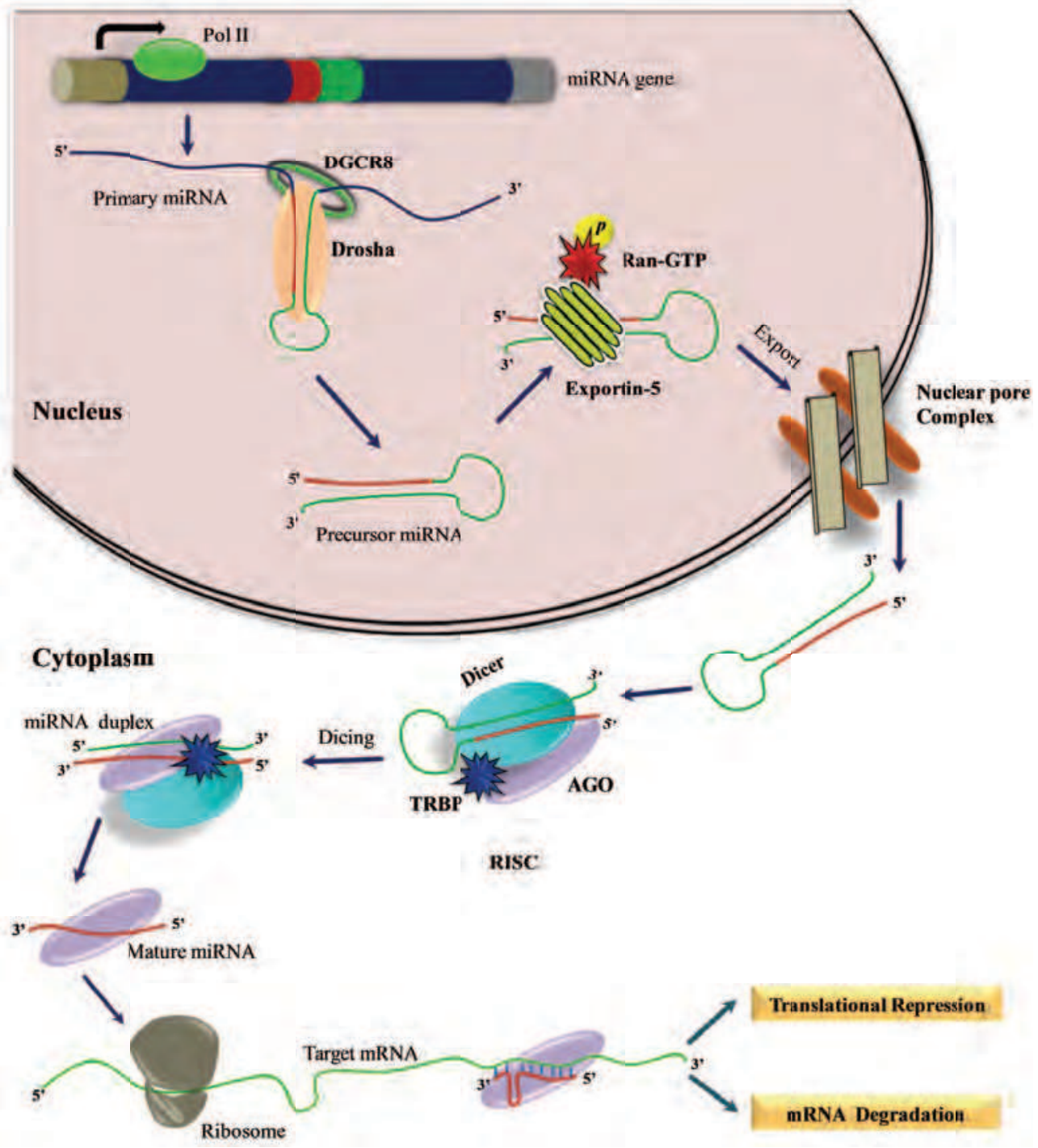

Fig. 1. Schematic representation of miRNA biogenesis pathway. 


\section{Methods to quantitate miRNA levels}

An array of techniques and methodologies can be employed to quantify the levels of miRNAs both absolutely and relatively. These include northern blotting, microarrays, bead based arrays and quantitative real-time RT-PCR. Northern blotting is a low throughput technique as well as less sensitive requiring more input RNA but it can be used to detect both mature and pre-miRNA molecules. Genome wide miRNA expression studies are chiefly carried out by employing various microarray platforms provided by different private firms and are based on different concepts.

Microarray based detection involves a set of oligonucleotide probes which are spotted on a glass slide and can capture the small molecule RNAs like miRNAs, if allowed to hybridize under optimum conditions. Availability of high density microarrays, where one can spot several thousands of oligonucleotide probes, provides the liberty to detect hundreds of miRNAs simultaneously on a single slide. Agilent Inc (http://www.agilent.com/) has developed a microarray based application for studying miRNAs that combines a unique miRNA direct labeling method with an innovative probe design and established highperformance SurePrint inkjet synthesis technology. miRNAs being small in size have an inherent limitation to hybridize at a single melting temperature (Tm) without compromising the sensitivity and specificity of the method. This problem has been overcome by employing locked nucleic acids (LNAs) developed by Exiqon Inc (http://www.exiqon.com/). Locked nucleic acids differ from regular nucleic acids by a methylene bridge between 2 ' oxygen atom and the $4^{\prime}$ carbon atom of the ribose moiety. LNA monomers increase the Tm of the nucleic acid duplex by $2-10^{\circ} \mathrm{C}$ and hence by adjusting the number of LNA units in the oligonucleotide, the hybridization can be carried out at a higher temperature thereby increasing the specificity.

Bead-based arrays also allow simultaneous quantification of hundreds of miRNAs (Luminex Inc). This methodology also uses oligonucleotide probes which are coupled to carboxylated polystyrene microspheres that incorporate variable mixtures of two fluorescent dyes hence allows the detection of each microsphere with a distinct color by a flow cytometer. Each microsphere is coupled with oligonucleotide molecules that are specific for a particular miRNA. The method involves RNA extraction, followed by biotinylation and finally hybridization. The microspheres are washed, incubated with streptavidin-phycoerythrin, and analyzed on a Luminex analyzer. The analyzer identifies the fluorescent microsphere as well as quantitates the intensity of the streptavidin-phycoerythrin fluorescence, resulting in the identification of miRNAs present in the sample.

Expression analysis is more commonly performed by quantitative real-time RT-PCR. Quantification of mature miRNAs usually involves reverse transcription of the miRNA with a stem-loop primer. miRNA specific cDNA is then used in the real-time PCR reaction. A mixture of forward and reverse primers and a dual-labeled probe (TaqMan ${ }^{\circledR}$ ) are used to amplify and detect the specific cDNA (Life Technologies Corp.). The probe has a reporter dye on the $5^{\prime}$ end and a quencher on the $3^{\prime}$ end. If the target sequence is present during the PCR, the probe binds to the target sequence. During the extension stage of the PCR cycle, the reporter dye is released by the $5^{\prime}$ exonuclease activity of Taq polymerase, and because the reporter and quencher have been separated, the fluorescence from the reporter dye is detected. A variation of quantitative real time RT-PCR using SybrGreen based chemistry has also been employed (System Biosciences, Qiagen etc.). The technique involves cDNA synthesis for the miRNA pool followed by real time PCR using a miRNA specific forward primer and a reverse primer essentially derived from RT primer. 


\section{4. miRNA regulation in glioma}

Since the initial reports by Ciafre et al and Chan et al, there is an increasing body of literature accumulated on the role of miRNAs in glioma development (Table. 1) (Chan et al., 2005; Ciafre et al., 2005). Various miRNA profiling studies carried out in glioma tissue samples identified differentially regulated miRNAs in gliomas. Several studies also reported the indepth investigation of specific miRNAs in glioma cells including functional characterization to understand the effects of regulation of miRNA in glioma cells with respect to various transformation related phenotypes like cell proliferation, cell death, migration and invasion. Many of these studies also included the prediction and experimental validation of targets of miRNA by using various accepted techniques.

\subsection{Large scale expression profiling of miRNAs in glioma}

The first report of expression profiling of large number of miRNAs in glioma was almost simultaneously reported by two groups (Chan et al., 2005; Ciafre et al., 2005). Ciafre et al used a microarray chip to profile the expression of 245 miRNAs in GBM tissue samples (Ciafre et al., 2005). The samples included nine GBM tissue samples, matched adjacent peripheral region as normal brain tissue and glioma cell lines. With the additional validation by northern blotting, this study identified miR-221 as GBM upregulated and miR-128, miR-181a, miR-181b and miR-181c as GBM downregulated miRNAs. Chan et al analyzed the expression of 180 mammalian miRNAs using a nylon membrane based oligonucleotide array in three primary high-grade gliomas and eight non-neoplastic fetal and adult brain tissues. They found miR-21 as the most upregulated miRNA in high grade gliomas among a total eight miRNAs (Chan et al., 2005). Silber et al analyzed the expression of 192 miRNAs by quantitative RT-qPCR with four AAs, four GBMs and four Glioses (as normal brain tissues) and found that 35 miRNAs were significantly deregulated in AA or GBM tumours (Silber et al., 2008). Thirteen (37\%) of these miRNAs were differentially expressed in both AA and GBM in comparison to glioses, $16(45 \%)$ were differentially expressed only in GBM tumours and $6(17 \%)$ were differentially expressed only in AA tumours. Another study carried out microarray based expression profiling of $245 \mathrm{miRNAs}$ and found that eleven miRNAs were downregulated and eight miRNAs were upregulated in GBM (Godlewski et al., 2008). Malzkorn et al analyzed the expression of 157 miRNAs in four glioma patients who had the progression of grade II to grade IV by real time RT-PCR (Malzkorn et al.). This revealed the differential expression of 14 miRNAs - of which, 12 miRNAs (miR-9, miR-15a, miR-16, miR-17, miR-19a, miR20a, miR-21, miR-25, miR-28, miR-130b, miR-140 and miR-210) were upregulated during progression whereas 2 (miR-184 and miR-328) of them were downregulated (Malzkorn et al.). The authors validated the expression of selected miRNAs (miR-16, miR-17, miR-19a, miR-20a, miR-140 and miR-184) in an independent set of low grade and secondary GBM samples thus confirming the grade associated regulation of these miRNAs. Modulation of miR-17 and miR-328 in glioma cell lines revealed an important role of these miRNAs in glioma cell proliferation, apoptosis and cell invasion phenotypes (Malzkorn et al.).

While the above studies were relatively small, investigating couple of hundred miRNAs and used fewer tissue samples, Rao et al recently profiled the expression of 756 human miRNAs in malignant astrocytoma- AA $(n=13)$ and GBM $(n=26)$, along with the normal brain samples $(n=7)$ and found that 55 miRNAs were upregulated and 29 miRNAs were downregulated in malignant astrocytomas in comparison to normal brain samples 


\begin{tabular}{|c|c|c|c|c|c|c|c|c|c|c|c|c|c|c|c|c|}
\hline 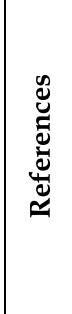 & 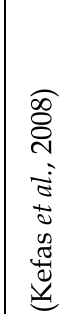 & 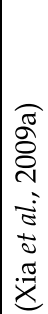 & 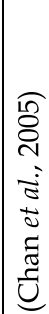 & 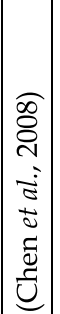 & 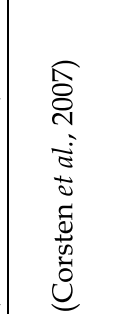 & 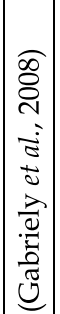 & 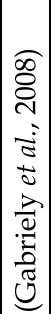 & 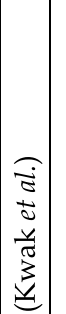 & 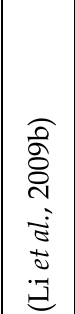 & 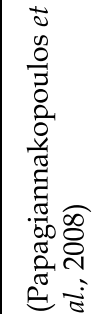 & 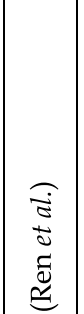 & 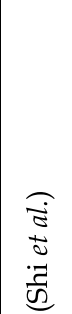 & 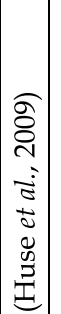 & 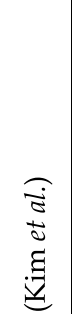 & 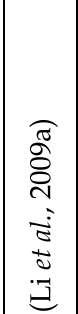 & 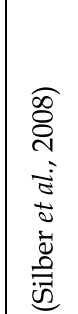 \\
\hline 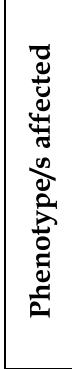 & 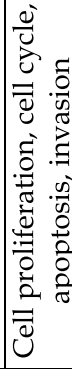 & 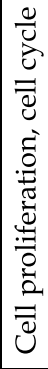 & 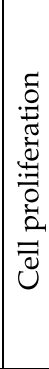 & \begin{tabular}{|l} 
\\
.0 \\
0 \\
0 \\
0 \\
0 \\
0 \\
0 \\
$\frac{2}{4}$ \\
\end{tabular} & 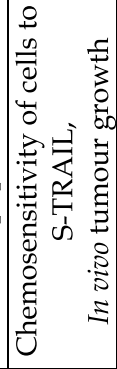 & 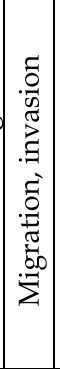 & 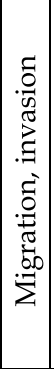 & 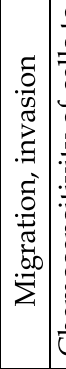 & 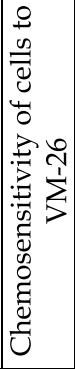 & 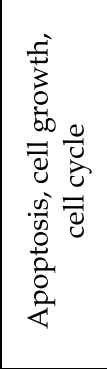 & 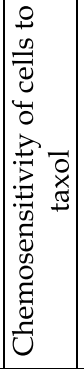 & 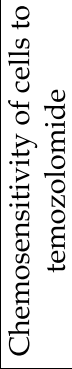 & 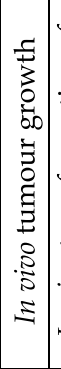 & 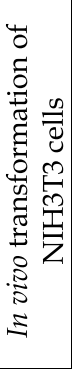 & 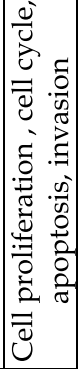 & 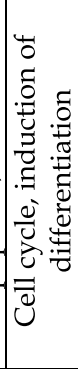 \\
\hline 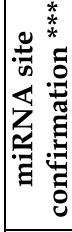 & $\stackrel{\infty}{\infty}$ & $\stackrel{\infty}{\infty}$ & I & I & ' & $\stackrel{\mathscr{D}}{\stackrel{\gamma}{ }}$ & zे & $\stackrel{\infty}{\varnothing}$ & $\stackrel{\otimes}{\otimes}$ & $\stackrel{\circ}{z}$ & & ' & $\stackrel{\infty}{\infty}$ & $\stackrel{\infty}{\stackrel{D}{~}}$ & $\stackrel{\infty}{D}$ & $\stackrel{\infty}{\infty}$ \\
\hline 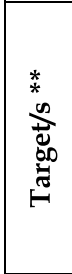 & 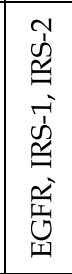 & $\underset{\mathrm{U}}{\tilde{I}}$ & ' & 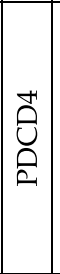 & 1 & 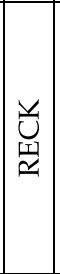 & $\sum_{i}^{\infty}$ & 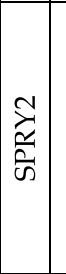 & 预 & 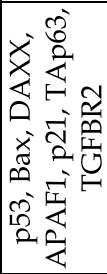 & & 1 & $\begin{array}{l}z \\
\text { Z } \\
\text { 至 } \\
a\end{array}$ & 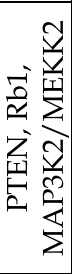 & 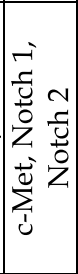 & $\stackrel{\vartheta}{\stackrel{\theta}{\theta}}$ \\
\hline 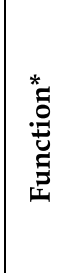 & 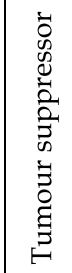 & 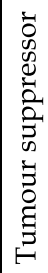 & & & & & & 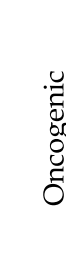 & 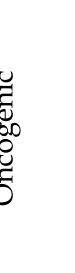 & & & & & 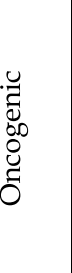 & 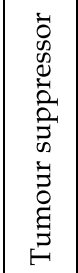 & 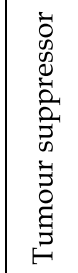 \\
\hline 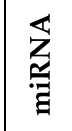 & 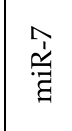 & 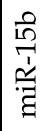 & & & & & & $\begin{array}{l}\tilde{N} \\
\stackrel{\tilde{a}}{\vec{g}}\end{array}$ & $\begin{array}{l}\bar{y} \\
\frac{y}{z} \\
\frac{a}{z}\end{array}$ & & & & & 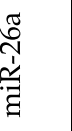 & 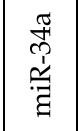 & 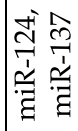 \\
\hline 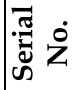 & $r$ & $N$ & & & & & & क & $n$ & & & & & + & 10 & 0 \\
\hline
\end{tabular}




\begin{tabular}{|c|c|c|c|c|c|}
\hline $\begin{array}{l}\text { Serial } \\
\text { No. }\end{array}$ & miRNA & Function* & Target/s ** & $\begin{array}{c}\text { miRNA site } \\
\text { confirmation } * * *\end{array}$ & Phenotype/s affec \\
\hline \multirow{2}{*}{7} & \multirow{2}{*}{ miR-125b } & Tumour suppressor & CDK6 and CDC25 & No & Proliferation, cell \\
\hline & & Oncogenic & BMF1 & No & Cell viability, apop \\
\hline 8 & miR-146b & Tumour suppressor & EGFR & Yes & Invasion, migrat \\
\hline \multirow[t]{2}{*}{9} & \multirow[t]{2}{*}{ miR-128 } & \multirow[t]{2}{*}{ Tumour suppressor } & Bmi1 & Yes & $\begin{array}{c}\text { In vivo tumour gro } \\
\text { Neurosphere formati } \\
\text { proliferation }\end{array}$ \\
\hline & & & E2F3a & Yes & Cell proliferatio \\
\hline 10 & miR-181a/b & Tumour suppressor & - & - & $\begin{array}{l}\text { Proliferation, apop } \\
\text { invasion, anchor } \\
\text { independent grov }\end{array}$ \\
\hline 11 & miR-218 & Tumour suppressor & IKK-B & Yes & Migration, invasi \\
\hline \multirow{2}{*}{12} & \multirow{2}{*}{$\mathrm{miR}-221 / 222$} & \multirow{2}{*}{ Oncogenic } & p27, p57 & Yes & Cell cycle \\
\hline & & & PUMA & Yes & Apoptosis \\
\hline 13 & miR-296 & Oncogenic & HGS & Yes & $\begin{array}{l}\text { Endothelial tubu } \\
\text { formation, migration, } \\
\text { tumour neovasculari }\end{array}$ \\
\hline 14 & $\operatorname{miR}-451$ & Tumour suppressor & - & - & $\begin{array}{r}\text { Cell proliferation, ce } \\
\text { apoptosis, invasi }\end{array}$ \\
\hline
\end{tabular}

* miRNAs are classified to have 'oncogenic' or 'tumour suppressor' function based on their pro-tum property respectively.

** miRNA targets are identified based on the reduction in the protein level with miRNA overexpres in the reporter activity of the 3'-UTR reporter.

*** miRNA site confirmation indicates whether the predicted miRNA binding site is confirmed by si abolishes the effects of miRNA overexpression on the reporter activity.

Table 1. Characterization of the role of miRNAs in glioma 
(Rao et al.). Besides the validation of several deregulated miRNAs, this study also developed a 23 miRNA expression signature that could distinguish between AA and GBM with high accuracy.

\subsection{Specific miRNAs in glioma development \\ 4.2.1 miR-21}

miR-21 is found to be upregulated in different kinds of human cancers including glioma. miR-21, being one of the well studied miRNAs in cancer, is shown to have oncogenic roles by targeting multiple tumour suppressors in human cancers. The expression profiling of miRNAs in glioblastoma tissues as well as in glioma cell lines identified miR-21 as an upregulated miRNA in glioma (Chan et al., 2005). Subsequently, the over expression of miR21 in gliomas was confirmed by several studies (Ciafre et al., 2005; Conti et al., 2009; Gabriely et al., 2008); (Papagiannakopoulos et al., 2008; Rao et al., ; Silber et al., 2008).

Modulation of miRNA levels in glioma cell lines indicated that miR-21 had pro-proliferative (Chan et al., 2005; Papagiannakopoulos et al., 2008; Zhou et al.) and anti-apoptotic effects (Chan et al., 2005; Papagiannakopoulos et al., 2008). miR-21 also affected cell cycle progression (Papagiannakopoulos et al., 2008) and the tumour growth in vivo (Corsten et al., 2007; Zhou et al.). miR-21 was also shown to modulate the chemosensitivity of glioma cells to many chemotherapeutic agents like doxorubicin, secretable tumour necrosis factor related apoptosis inducing ligand (S-TRAIL), taxol, VM-26 and temozolomide (Corsten et al., 2007; Li et al., 2009b; Papagiannakopoulos et al., 2008; Ren et al., ; Shi et al.). Papagiannakopoulos et al undertook systems biology approach to understand the role of miR-21 in glioblastoma development. The network analysis of bioinformatically predicted targets of miR-21 showed that the over expressed miRNA was linked to three major cancer pathways - p53, TGF beta and mitochondrial apoptosis pathways. The knock down of miR-21 in glioma cell line upregulated many tumour suppressor proteins like p53, Bax, DAXX, APAF1, p21, TAp63 and TGFBR2 (Papagiannakopoulos et al., 2008).

Since miR-21 is one of the most upregulated miRNAs, efforts are being made to identify its direct targets. While the tumour suppressor proteins like PTEN (phosphatase and tensin homolog), TPM1 (Tropomyosin 1) and maspin were well characterized as direct targets of miR-21 in other cancers, they were not validated in glioblastoma (Novakova et al., 2009). Nevertheless, it was shown that the anti-apoptotic nature of the miR-21 can be partly attributed to its target PDCD4 (programmed cell death 4), as the over-expression of miR-21 inhibited PDCD4-dependent apoptosis (Chen et al., 2008). In addition to its effects on growth, miR-21 also affected the property of invasion that causes infiltrating pattern of glioma growth in vivo (Gabriely et al., 2008). Using the target prediction algorithms and the transcript profiling, the matrix metalloprotease inhibitors, RECK and TIMP3 were identified as miR-21 targets thus substantiating a pro-invasion and -migration role for miR-21 (Gabriely et al., 2008). The promotion of invasion was also attributed to another target of miR-21, sprouty, the negative regulator of Ras/MAPK pathways (Kwak et al.).

\subsection{2 miR-26a}

The upregulation of miR-26a and its consequence on cellular pathways leading to the protumorigenic effects are well studied in glioblastoma. In GBM, PTEN expression was found to be directly regulated by miR-26a (Huse et al., 2009). The miR-26a locus was found to be 
amplified and it was often associated with the monoallelic loss of PTEN suggesting that the residual PTEN transcript made in PTEN +/- cases was nullified by miR-26a, analogous to loss of heterozygosity. Using The Cancer Genome Atlas (TCGA) data, authors demonstrated that, in patients with monoallelic PTEN deletion, amplification of miR-26a locus served as another mechanism to inactivate PTEN. In the mouse glioma model, miR-26a mediated PTEN repression enhanced tumour formation and substituted the loss of heterozygosity of PTEN locus. Further studies carried out by Kim et al demonstrated that hsa-miR-26a is a cooperating component of a frequently occurring oncogenic amplicon containing CDK4 and CENTG1 which regulate the RB1 and PI3 kinase/AKT pathways (Kim et al.). By integrating the TCGA data for copy number, mRNA, miRNA, and DNA methylation, they identified PTEN, RB1, and MAP3K2/MEKK2 to be the functional targets of miR-26a in GBM. They also demonstrated that miR-26a alone could transform NIH3T3 cells and promote the growth of glioma cells in vivo by directly regulating the expressions of PTEN, RB1, and MAP3K2/MEKK2. This led to the increased AKT activation and decreased c-JUN Nterminal kinase-dependent apoptosis resulting in increased proliferation. In both PTENpositive and PTEN-deficient glioma cells, the over expression of miR-26a induced the tumour growth in vivo, and it was further increased if the cells were over expressing CDK4 or CENTG1. GBM patients with the amplification of miR-26a-CDK4 locus had markedly decreased survival. Thus, the amplification of hsa-miR-26a, CDK4, and CENTG1 increased the aggressiveness of the tumour by cooperately regulating RB1, PI3K/AKT, and JNK pathways (Kim et al.).

\subsection{3 miR-221 and miR-222}

The comprehensive analysis of miRNA expression during serum stimulation of quiescent T98 glioblastoma cells revealed that miR-221 and miR-222 were upregulated as cells progressed beyond the G1-S phase transition (Medina et al., 2008). miR-221 and miR-222 were found to target CDK inhibitors- p27 and p57 and prevent quiescence when elevated during growth factor deprivation and induced cell death by causing precocious S-phase entry. Further, the inhibition of the expression of these miRNAs in glioma cell lines led to the increased apoptosis (Zhang et al.). The effect of the miRNA on apoptosis was also attributed to its ability to target pro-apoptotic gene PUMA. The over expression of PUMA reversed the phenotypes caused by the over expression of the miRNAs. In tumour xenograft model, inhibition of miR-221/222 reduced the tumour volume compared to the scramble control along with increased apoptosis, reduced Ki-67 staining, increased PUMA and Bax and reduced Bcl-2 expression. In human glioma tissue samples, the down regulation of PUMA negatively correlated with the upregulation of miR-221/222 suggesting the importance of the regulation in the clinical samples.

\subsection{4 miR-296}

The profiling of miRNAs upon co-culturing human brain endothelial cells (HBMVECs) with human glioma cell line U87 identified that miR-296 is the only upregulated miRNA (Wurdinger et al., 2008). The miR-296 expression also increased in HBMVECs upon treatment with angiogenic factors like EGM, VEGF and EGF. Treatment of HBMVECs with VEGF resulted in the tubule formation, increased the migration distance and also increased the miR-296 level in dose dependent manner. Treatment of cells with miR-296 
inhibitors led to decreased tubule formation and migration distance. On the other hand, the overexpression of the miRNA led to increased tubule formation. The target prediction algorithms and subsequently, experimental evidences suggested that HGS, hepatocyte growth factor regulated receptor kinase substrate, to be the target of the miR-296. HGS was previously known to regulate the levels of VEGFR2 and PDGFR $\beta$ receptors. These studies suggested that the miR-296 in HBMVECs target HGS with the resultant upregulation of VEGFR2 and PDGFR $\beta$ receptors thereby promoting angiogenesis. To understand the in vivo role of miR-296 in tumour angiogenesis, nude mice-bearing subcutaneous U87 xenografts were injected with cholesterol-conjugated miR-296 antisense oligonucleotide. The quantification of tumour neovascularization revealed that the mice injected with antisense miR-296 showed reduced tumour neovascularization as compared to the mice injected with the mismatch control. The endothelial cells derived from human glioma showed upregulation of miR-296 compared to the normal brain with concomitant downregulation of HGS and the upregulation of the receptors-VEGFR2 and PDGFR $\beta$. These observations demonstrate the importance of miR-296 in the angiogenesis of malignant glioma.

\subsection{5 miR-7}

miR-7 was found to be a tumour suppressor miRNA and was downregulated in glioma compared to the normal brain. The in vivo experiments suggested that re-expression of miR7 into glioma cells decreased the cell count, invasion and increased apoptosis (Kefas et al., 2008). Oncogenic proteins, EGFR, IRS-1 and IRS-2 were identified as the targets of miR-7. The miR-7 inhibited the Akt pathway but this inhibition could not be rescued by the over expression of wild type or the mutant EGFR. Further investigation revealed that the impaired processing as the mechanism of downregulation of miR-7 in glioma. This study unraveled the tumour suppressor property of the miR-7 by targeting the receptors-EGFR, IRS1 and IRS2, thereby counteracting the pro-proliferative effect of the pathway.

\subsection{6 miR-34a}

miR-34a was found to act as a tumour suppressor in glioma by targeting multiple oncogenes like c-Met, Notch1, Notch2 and CDK6 (Li et al., 2009a). The miR-34a expression in glioma tissues was downregulated compared to the normal brain. As the earlier reports suggested that miR-34a is a target of p53, authors analyzed the expression of the miRNA in cell lines with wild type p53 and with mutant p53 and found that the expression of miR-34a in mutant p53 is lower compared to that in those cell lines bearing wild type p53. In the glioma tissues, the c-Met and the miR-34a expression negatively correlated suggesting the miRNA mediated regulation of c-Met in glioma. To assess the effect of the miRNA on cell proliferation, cell cycle and apoptosis, the miR-34a was over expressed in different cell lines, along with human astrocytes. The miR-34a over expression reduced the proliferation, the increased G1/G0 population only in cancer cell lines, but not in normal human astrocytes. The invasion of the cells also decreased with miR-34a over expression. The over expression of c-met partially but significantly rescued the effect of miR-34a over expression on cell cycle where as the over expression of Notch1 and Notch2 rescued miR-34a effect on apoptosis. In vivo studies suggested that the miR-34a over expression in U87 cells led to the development of much smaller tumours compared to the control U87 cells. 


\subsection{7 $\mathrm{miR}-124$ and $\mathrm{miR}-137$}

Expression profiling revealed that the levels of miR-124 and miR-137 were significantly decreased in AA and GBM relative to non-neoplastic brain tissue (Silber et al., 2008). During the differentiation of cultured mouse neural stem cells following growth factor withdrawal, the levels of these miRNAs were increased. The re-expression of these miRNAs with the DNA methylation inhibitor treatment suggested a possible regulation of these miRNAs by epigenetic phenomenon like methylation. The over expression of miR-124 or miR-137 induced morphological changes and also induced differentiation marker profile. Further studies identified that miR-124 and miR-137 target CDK6 resulting in reduced phosphorylated RB and G1 cell cycle arrest.

\subsection{8 miR-128}

The expression analysis revealed that miR-128 was downregulated miRNAs in glioma (Godlewski et al., 2008; Zhang et al., 2009). Over expression of the miRNA led to the decreased cell proliferation and tumour growth in vivo (Godlewski et al., 2008) suggesting the tumour suppressor nature of the miRNA. miR-128 was shown to target Bmi1 (B lymphoma mouse Moloney leukemia virus insertion region) oncogene. In the GBM tumours, the downregulation of miR-128 correlated with the corresponding upregulation of Bmi1 oncogene. The miR-128 over expression caused the reduction of $\mathrm{H} 3 \mathrm{~K}$ trimethylation and pAkt and also increased p21 suggesting that the cellular pathways regulated by Bmi1 were altered with miRNA over expression leading to the decreased neurosphere formation. miR-128 was also shown to directly target E2F3a transcription factor generally upregulated in glioma. The over expression of E2F3a rescued the cell growth inhibition caused by the over expression of the miR-128 (Zhang et al., 2009).

\subsection{9 miR-218}

miR-218 had reduced expression in glioma cell lines compared to the normal brain (Song et al.). The over expression of the miRNA in glioma cell lines resulted in the decreased migration and invasion of the glioma cells with concomitant decreased MMP-9 activity. The over expression of miR-218 in glioma cell lines also resulted in the downregulation of the targets of NF-kB pathway suggesting the role of the miRNA in regulating the pathway. Using the target prediction algorithms, authors identified that miR-218 targeted IKK- $\beta$ thereby regulating NF-kB pathway (Song et al.).

\subsubsection{0 miR-125b}

The expression of miR-125, a previously identified brain-specific miRNA, was found to be reduced when glioma cells were treated with all trans retinoic acid (ATRA) (Xia et al., 2009c). The over expression of miR-125b increased the cell viability in the presence or in the absence of ATRA along with the decrease in the apoptosis. The authors identified Bmf (Bcl-2 modifying factor) as the direct target of miR-125b. In contrast to this report, another study reported that the over expression of miR-125b in glioma cells led to the decreased cell growth and caused the reduced progression of the cells through cell cycle by downregulating the levels of CDK6 and CDC25 (Shi et al.). The study also reported that miR-125b was expressed at a higher level in CD133 positive glioma stem cells compared to the CD133 negative non-stem cell population. 


\subsubsection{1 miR-451}

miR-451 is one of the significantly downregulated miRNA in glioma compared to the normal brain (Nan et al.). The over expression of miR-451 in glioma cell lines led to decreased proliferation and invasion, in addition to Go/G1 arrest and apoptosis induction. The over expression of miR-451 reduced Akt, cyclinD1, MMP2, MMP9 and Bcl-2 also increased p27/kip levels suggesting the tumour suppressor nature of the miRNA. However, the above identified miR-451 targets have not been experimentally validated (Nan et al.).

\subsubsection{2 miR-15b}

The modulation of miR-15b in U87 and U118 glioma cell lines demonstrated that miR-15b affects the cell growth and the cell cycle progression (Xia et al., 2009a). The bioinformatics prediction identified cyclins- cyclin E1 and cyclin D to be the targets of miR-15b. The authors further demonstrated that cyclin E1 was indeed a direct target of miR-15b.

\subsubsection{3 miR-181a and miR-181b}

The miRNAs- miR-181a and miR-181b were found to be downregulated in glioma by multiple studies (Ciafre et al., 2005; Conti et al., 2009; Malzkorn et al.). The overexpression of these miRNAs in glioma cell lines resulted in the reduction of cell growth in vivo, anchorage independent growth, invasion and apoptosis (Shi et al., 2008).

\subsubsection{4 miR-146b}

The modulation of miR-146b in glioma cell line revealed that the migration and invasion were inhibited by miR-146b (Katakowski et al., ; Xia et al., 2009b). miR-146 was found to target MMP-16 which could potentially mediate the reduced migration and invasion seen upon miR-146b overexpression (Xia et al., 2009b). miR-146b was also shown to directly target EGFR (Katakowski et al).

\section{Applications of miRNA in glioma: accurate grading, better prognosis and novel therapeutic approaches}

The rationale for using miRNAs in glioma diagnosis and therapeutic modalities is as follows. The identification of differences in the genetic alterations in miRNA genes and miRNA transcript levels in tumour samples compared to normal tissues provides an automatic basis for their utility for glioma diagnosis, classification and prognosis. In fact, classification of multiple cancers based on the expression pattern of couple of hundred miRNAs was found to be more accurate than using expression of approximately 16,000 protein coding genes (Lu et al., 2005). Even though several miRNAs have been shown to be regulated in different grades of glioma, variation in the expression of individual miRNAs within a glioma grade suggests that distinct subgroups within each grade of glioma could be identified. It is likely that these subgroups may have different prognosis. miRNAs also appear to be an attractive target for therapeutic intervention because the aberrantly expressed miRNAs seem to play key role in the development of cancer and that correcting the miRNA deficiencies either by antagonizing or restoring miRNA function may offer a therapeutic gain (Bader et al.).

Although several miRNAs have been identified to be either upregulated or down regulated in glioblastoma, there were only few studies which particularly investigated the differential 
regulation of miRNAs between different grades of glioma. Real time RT-PCR analysis of several miRNAs in WHO grade II and grade IV samples which spontaneously progressed from the same set of patients identified twelve miRNAs (miR-9, miR-15a, miR-16, miR-17, miR-19a, miR-20a, miR-21, miR-25, miR-28, miR-130b, miR-140 and miR-210) to be upregulated and two miRNAs (miR-184 and miR-328) to be downregulated in grade IV tumours thus characterizing their role in progression (Malzkorn et al.). Analysis of miRNA microarray data by prediction analysis of microarrays (PAM), a 23 miRNA expression signature was identified that could distinguish GBM (grade IV) from AA (grade III) with an accuracy of $95 \%$ (Rao et al.).

While many studies identifying an individual miRNA or miRNA expression signatures predicting patient survival in several cancers like lung cancer, lymphocytic leukemia; lung adenocarcinoma, breast and pancreas cancers have been published, there are only a few reports with respect to glioblastoma. Expression profiling of 84 astrocytoma samples belonging to different grades and 20 normal adjacent tissue samples and subsequent multivariate Cox proportional hazard regression analysis and Kaplan-Meier survival analysis identified low expression of miR-181b or miR-106a or high expression of miR-21 to be associated with poor patient survival (Zhi et al.). Another recent study analysed the miRNA ( $n=304)$ expression data from a total of 222 GBM patients derived from The Cancer Genome Atlas (TCGA) data set using Cox proportional hazard regression analysis and identified a 10 miRNA expression signature that predicted survival (Srinivasan et al.). More importantly, using multivariate analysis along with patient age, the 10 miRNA expression signature was found to be an independent predictor of patient survival. In addition, the authors developed a risk score for each patient based on the expression of each of these ten miRNAs and subsequently showed that the risk score could segregate the patients into high and low risk groups with significantly different survival times. It was also interesting to note that the miRNA set included three miRNAs (miR-20a, miR-106a and miR-17-5p) that were protective and seven miRNAs (hsa-miR-31, hsa-miR-222, hsa-miR-148a, hsa-miR-221, hsa-miR-146b, hsa-miR-200b and hsa-miR-193a) that were risky with respect to their association between their expression and patient survival. The protective miRNAs were expressed at an elevated level in the low risk group compared to the high risk group and the risky miRNAs were expressed at a higher level in the high risk group than in the low risk group. It was suggested that the protective and risky nature of these miRNAs is indicative of their functions being either inhibitory or promoting, respectively, of various properties of cancer cells like proliferation, migration, invasion etc.

The use of miRNAs in cancer therapy involves two approaches. The first approach, which is rather more commonly followed, is to inhibit oncogenic miRNAs with a gain of function using miRNA antagonists. The second approach involves reintroduction of a tumour suppressor miRNA with the resultant restoration of loss of function. An interesting view of the potential use of miRNA based cancer therapy is that targets that are considered to be undruggable could be targeted using miRNA approach. It was estimated that approximately only $20 \%$ of all human proteins are targetable by small molecules and inhibitory antibodies with the remaining $80 \%$ of genes coming under the category that are currently considered undruggable (Verdine, 2006). Since the RNA interference based approach using miRNAs can essentially target any human gene, this approach opens up a large number of potential targets. 
Several reports have shown the potential use of miRNA-based approach in glioma therapy as a proof-of-principle. miR-21 being one of the most upregulated miRNAs in gliomas, with anti-apoptotic and pro-invasive functions, appears to be an automatic choice for therapeutic interventions. Corsten et al studied the combined effect of inhibiting miR-21 and neural precursor cells (NPC)-mediated secretable variant of the cytotoxic agent tumour necrosis factor-related apoptosis inducing ligand (S-TRAIL) on glioma (U87) tumour growth using an intra-cranial glioma model using athymic mice (Corsten et al., 2007). The ability of U87 glioma cells to form tumour reduced significantly upon introduction of locked nucleic acid (LNA) antimiR-21 oligonucleotides. Further, simultaneous injection of NPCs expressing STRAIL along with miR-21 anti-miR treated U87 cells led to synergistic cytotoxicity with the complete elimination of tumour growth. In another study involving a tumour suppressor miRNA - miR-34a, its reintroduction into U87 glioma cells led to strong inhibition of tumour growth in xenograft models (Li et al., 2009a). miR-296, which belongs to the new family of angiomirs, which are proangiogenic, was found to be elevated in human primary brain microvascular endothelial cells upon co-culturing with U87 glioma cells (Wurdinger et al., 2008). Intravenous administration of miR-296 antagomirs into mice bearing subcutaneous U87 tumours resulted in significant reduction of angiogenesis in tumour xenografts suggesting the potential utility in glioma therapy.

\section{Conclusions}

miRNAs have generated a huge excitement in the field of cancer biology. During the last six years, several studies have established the regulation of miRNAs in glioma development, identified their targets and demonstrated their role in cell proliferation, apoptosis, cell cycle regulation, migration, invasion and angiogenesis. This indicates that defects in miRNA regulatory network appear to play a key role in glioblastoma pathogenesis. Many of these studies have clearly showed as a proof-of-concept, the utility of miRNA based approach in glioma diagnosis, grading, prognosis and therapy. However, additional studies are needed to confirm these findings for the eventual application in clinics. With total number of known human miRNAs is 1040 (Release 16, miRBase), one of important needs is to carry out more comprehensive analysis of all known miRNAs in large number of tumour tissue samples. Efforts towards identifying targets, their experimental validation and establishing the relationships with various altered molecular pathways in cancer are also very important. In addition to improved understanding of biology of glioma development and progression, miRNA study appears to provide newer improved methods useful for variety of purposes in clinical medicine. Like with other biomarkers, the use of miRNAs in glioma classification and prognosis requires more studies in large cohorts at independent laboratories. The use of miRNA based glioma therapy appears to be very highly promising. The development of better methods of in vivo delivery of miRNAs is likely to harness the utility of miRNA in glioma therapy.

\section{Acknowledgements}

This study was supported by a grant from DBT, Government of India. Infrastructural support by funding from ICMR, DBT, DST and UGC to MCB is acknowledged. SAR and ZN gratefully acknowledge SRF from CSIR. 


\section{References}

Bader AG, Brown D, Winkler M The promise of microRNA replacement therapy. Cancer Res 70: 7027-30.

Bohnsack MT, Czaplinski K, Gorlich D (2004). Exportin 5 is a RanGTP-dependent dsRNAbinding protein that mediates nuclear export of pre-miRNAs. RNA 10: 185-91.

Brueckner B, Stresemann C, Kuner R, Mund C, Musch T, Meister M et al (2007). The human let-7a-3 locus contains an epigenetically regulated microRNA gene with oncogenic function. Cancer Res 67: 1419-23.

Cai X, Hagedorn CH, Cullen BR (2004). Human microRNAs are processed from capped, polyadenylated transcripts that can also function as mRNAs. RNA 10: 1957-66.

Chan JA, Krichevsky AM, Kosik KS (2005). MicroRNA-21 is an antiapoptotic factor in human glioblastoma cells. Cancer Res 65: 6029-33.

Chen Y, Liu W, Chao T, Zhang Y, Yan X, Gong Y et al (2008). MicroRNA-21 down-regulates the expression of tumor suppressor PDCD4 in human glioblastoma cell T98G. Cancer Lett 272: 197-205.

Chendrimada TP, Gregory RI, Kumaraswamy E, Norman J, Cooch N, Nishikura K et al (2005). TRBP recruits the Dicer complex to Ago2 for microRNA processing and gene silencing. Nature 436: 740-4.

Ciafre SA, Galardi S, Mangiola A, Ferracin M, Liu CG, Sabatino G et al (2005). Extensive modulation of a set of microRNAs in primary glioblastoma. Biochem Biophys Res Commun 334: 1351-8.

Conti A, Aguennouz M, La Torre D, Tomasello C, Cardali S, Angileri FF et al (2009). miR-21 and 221 upregulation and miR-181b downregulation in human grade II-IV astrocytic tumors. J Neurooncol 93: 325-32.

Corsten MF, Miranda R, Kasmieh R, Krichevsky AM, Weissleder R, Shah K (2007). MicroRNA-21 knockdown disrupts glioma growth in vivo and displays synergistic cytotoxicity with neural precursor cell delivered S-TRAIL in human gliomas. Cancer Res 67: 8994-9000.

Davis BN, Hata A (2009). Regulation of MicroRNA Biogenesis: A miRiad of mechanisms. Cell Commun Signal 7: 18.

Davis BN, Hilyard AC, Lagna G, Hata A (2008). SMAD proteins control DROSHA-mediated microRNA maturation. Nature 454: 56-61.

Du T, Zamore PD (2005). microPrimer: the biogenesis and function of microRNA. Development 132: 4645-52.

Gabriely G, Wurdinger T, Kesari S, Esau CC, Burchard J, Linsley PS et al (2008). MicroRNA 21 promotes glioma invasion by targeting matrix metalloproteinase regulators. $\mathrm{Mol}$ Cell Biol 28: 5369-80.

Godlewski J, Nowicki MO, Bronisz A, Williams S, Otsuki A, Nuovo G et al (2008). Targeting of the Bmi-1 oncogene/stem cell renewal factor by microRNA-128 inhibits glioma proliferation and self-renewal. Cancer Res 68: 9125-30.

Gregory RI, Chendrimada TP, Cooch N, Shiekhattar R (2005). Human RISC couples microRNA biogenesis and posttranscriptional gene silencing. Cell 123: 631-40. 
Gregory RI, Yan KP, Amuthan G, Chendrimada T, Doratotaj B, Cooch N et al (2004). The Microprocessor complex mediates the genesis of microRNAs. Nature 432: 235-40.

He L, He X, Lowe SW, Hannon GJ (2007). microRNAs join the p53 network--another piece in the tumour-suppression puzzle. Nat Rev Cancer 7: 819-22.

Huse JT, Brennan C, Hambardzumyan D, Wee B, Pena J, Rouhanifard SH et al (2009). The PTEN-regulating microRNA miR-26a is amplified in high-grade glioma and facilitates gliomagenesis in vivo. Genes Dev 23: 1327-37.

Hutvagner G, Zamore PD (2002). A microRNA in a multiple-turnover RNAi enzyme complex. Science 297: 2056-60.

Katakowski M, Zheng X, Jiang F, Rogers T, Szalad A, Chopp M MiR-146b-5p suppresses EGFR expression and reduces in vitro migration and invasion of glioma. Cancer Invest 28: 1024-30.

Kefas B, Godlewski J, Comeau L, Li Y, Abounader R, Hawkinson M et al (2008). microRNA-7 inhibits the epidermal growth factor receptor and the Akt pathway and is downregulated in glioblastoma. Cancer Res 68: 3566-72.

Kim H, Huang W, Jiang X, Pennicooke B, Park PJ, Johnson MD Integrative genome analysis reveals an oncomir/oncogene cluster regulating glioblastoma survivorship. Proc Natl Acad Sci U S A 107: 2183-8.

Kim VN (2005). MicroRNA biogenesis: coordinated cropping and dicing. Nat Rev Mol Cell Biol 6: 376-85.

Kwak HJ, Kim YJ, Chun KR, Woo YM, Park SJ, Jeong JA et al Downregulation of Spry2 by miR-21 triggers malignancy in human gliomas. Oncogene.

Landthaler M, Yalcin A, Tuschl T (2004). The human DiGeorge syndrome critical region gene 8 and Its $\mathrm{D}$. melanogaster homolog are required for miRNA biogenesis. Curr Biol 14: 2162-7.

Lee RC, Feinbaum RL, Ambros V (1993). The C. elegans heterochronic gene lin-4 encodes small RNAs with antisense complementarity to lin-14. Cell 75: 843-54.

Lee Y, Hur I, Park SY, Kim YK, Suh MR, Kim VN (2006). The role of PACT in the RNA silencing pathway. EMBO J 25: 522-32.

Lee Y, Kim M, Han J, Yeom KH, Lee S, Baek SH et al (2004). MicroRNA genes are transcribed by RNA polymerase II. EMBO J 23: 4051-60.

Lewis BP, Burge CB, Bartel DP (2005). Conserved seed pairing, often flanked by adenosines, indicates that thousands of human genes are microRNA targets. Cell 120: $15-20$.

Li Y, Guessous F, Zhang Y, Dipierro C, Kefas B, Johnson E et al (2009a). MicroRNA-34a inhibits glioblastoma growth by targeting multiple oncogenes. Cancer Res 69: 756976.

Li Y, Li W, Yang Y, Lu Y, He C, Hu G et al (2009b). MicroRNA-21 targets LRRFIP1 and contributes to VM-26 resistance in glioblastoma multiforme. Brain Res 1286: 13-8.

Liu J, Carmell MA, Rivas FV, Marsden CG, Thomson JM, Song JJ et al (2004). Argonaute2 is the catalytic engine of mammalian RNAi. Science 305: 1437-41. 
Lu J, Getz G, Miska EA, Alvarez-Saavedra E, Lamb J, Peck D et al (2005). MicroRNA expression profiles classify human cancers. Nature 435: 834-8.

Lujambio A, Calin GA, Villanueva A, Ropero S, Sanchez-Cespedes M, Blanco D et al (2008). A microRNA DNA methylation signature for human cancer metastasis. Proc Natl Acad Sci U S A 105: 13556-61.

Lund E, Guttinger S, Calado A, Dahlberg JE, Kutay U (2004). Nuclear export of microRNA precursors. Science 303: 95-8.

Malzkorn B, Wolter M, Liesenberg F, Grzendowski M, Stuhler K, Meyer HE et al Identification and functional characterization of microRNAs involved in the malignant progression of gliomas. Brain Pathol 20: 539-50.

Maniataki E, Mourelatos Z (2005). A human, ATP-independent, RISC assembly machine fueled by pre-miRNA. Genes Dev 19: 2979-90.

Medina R, Zaidi SK, Liu CG, Stein JL, van Wijnen AJ, Croce CM et al (2008). MicroRNAs 221 and 222 bypass quiescence and compromise cell survival. Cancer Res 68: 277380.

Meister G, Landthaler M, Patkaniowska A, Dorsett Y, Teng G, Tuschl T (2004). Human Argonaute2 mediates RNA cleavage targeted by miRNAs and siRNAs. Mol Cell 15: 185-97.

Nan Y, Han L, Zhang A, Wang G, Jia Z, Yang Y et al MiRNA-451 plays a role as tumor suppressor in human glioma cells. Brain Res 1359: 14-21.

Novakova J, Slaby O, Vyzula R, Michalek J (2009). MicroRNA involvement in glioblastoma pathogenesis. Biochem Biophys Res Commun 386: 1-5.

Papagiannakopoulos T, Shapiro A, Kosik KS (2008). MicroRNA-21 targets a network of key tumor-suppressive pathways in glioblastoma cells. Cancer Res 68: 8164-72.

Rao SA, Santosh V, Somasundaram K Genome-wide expression profiling identifies deregulated miRNAs in malignant astrocytoma. Mod Pathol 23: 1404-17.

Ren Y, Zhou X, Mei M, Yuan XB, Han L, Wang GX et al MicroRNA-21 inhibitor sensitizes human glioblastoma cells U251 (PTEN-mutant) and LN229 (PTEN-wild type) to taxol. BMC Cancer 10: 27.

Ruby JG, Jan C, Player C, Axtell MJ, Lee W, Nusbaum C et al (2006). Large-scale sequencing reveals 21U-RNAs and additional microRNAs and endogenous siRNAs in C. elegans. Cell 127: 1193-207.

Ruby JG, Jan CH, Bartel DP (2007). Intronic microRNA precursors that bypass Drosha processing. Nature 448: 83-6.

Shi L, Chen J, Yang J, Pan T, Zhang S, Wang Z MiR-21 protected human glioblastoma U87MG cells from chemotherapeutic drug temozolomide induced apoptosis by decreasing Bax/Bcl-2 ratio and caspase-3 activity. Brain Res 1352: 255-64.

Shi L, Cheng Z, Zhang J, Li R, Zhao P, Fu Z et al (2008). hsa-mir-181a and hsa-mir-181b function as tumor suppressors in human glioma cells. Brain Res 1236: 185-93.

Shi L, Zhang J, Pan T, Zhou J, Gong W, Liu N et al MiR-125b is critical for the suppression of human U251 glioma stem cell proliferation. Brain Res 1312: 120-6.

Silber J, Lim DA, Petritsch C, Persson AI, Maunakea AK, Yu M et al (2008). miR-124 and miR-137 inhibit proliferation of glioblastoma multiforme cells and induce differentiation of brain tumor stem cells. BMC Med 6: 14 . 
Song L, Huang Q, Chen K, Liu L, Lin C, Dai T et al miR-218 inhibits the invasive ability of glioma cells by direct downregulation of IKK-beta. Biochem Biophys Res Commun 402: 135-40.

Srinivasan S, Patric IR, Somasundaram K A Ten-microRNA Expression Signature Predicts Survival in Glioblastoma. PLoS One 6: e17438.

Verdine GL (2006). Drugging the "undruggable". Harvey Lect 102: 1-15.

Wurdinger T, Tannous BA, Saydam O, Skog J, Grau S, Soutschek J et al (2008). miR-296 regulates growth factor receptor overexpression in angiogenic endothelial cells. Cancer Cell 14: 382-93.

Xia H, Qi Y, Ng SS, Chen X, Chen S, Fang M et al (2009a). MicroRNA-15b regulates cell cycle progression by targeting cyclins in glioma cells. Biochem Biophys Res Commun 380: 205-10.

Xia H, Qi Y, Ng SS, Chen X, Li D, Chen S et al (2009b). microRNA-146b inhibits glioma cell migration and invasion by targeting MMPs. Brain Res 1269: 158-65.

Xia HF, He TZ, Liu CM, Cui Y, Song PP, Jin XH et al (2009c). MiR-125b expression affects the proliferation and apoptosis of human glioma cells by targeting Bmf. Cell Physiol Biochem 23: 347-58.

Yi R, Qin Y, Macara IG, Cullen BR (2003). Exportin-5 mediates the nuclear export of premicroRNAs and short hairpin RNAs. Genes Dev 17: 3011-6.

Zhang CZ, Zhang JX, Zhang AL, Shi ZD, Han L, Jia ZF et al MiR-221 and miR-222 target PUMA to induce cell survival in glioblastoma. Mol Cancer 9: 229.

Zhang Y, Chao T, Li R, Liu W, Chen Y, Yan X et al (2009). MicroRNA-128 inhibits glioma cells proliferation by targeting transcription factor E2F3a. J Mol Med 87: 43-51.

Zhi F, Chen X, Wang S, Xia X, Shi Y, Guan W et al The use of hsa-miR-21, hsa-miR-181b and hsa-miR-106a as prognostic indicators of astrocytoma. Eur J Cancer 46: 16409.

Zhou X, Ren Y, Moore L, Mei M, You Y, Xu P et al Downregulation of miR-21 inhibits EGFR pathway and suppresses the growth of human glioblastoma cells independent of PTEN status. Lab Invest 90: 144-55. 


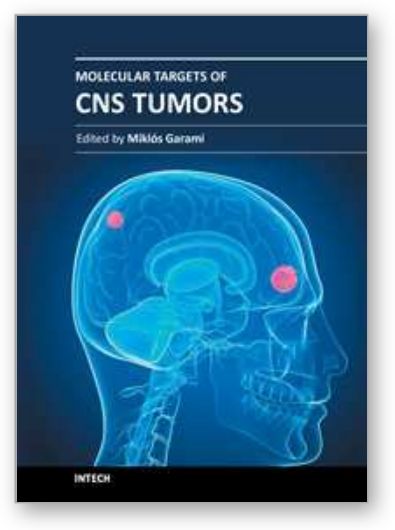

\author{
Molecular Targets of CNS Tumors \\ Edited by Dr. Miklos Garami
}

ISBN 978-953-307-736-9

Hard cover, 674 pages

Publisher InTech

Published online 22, September, 2011

Published in print edition September, 2011

Molecular Targets of CNS Tumors is a selected review of Central Nervous System (CNS) tumors with particular emphasis on signaling pathway of the most common CNS tumor types. To develop drugs which specifically attack the cancer cells requires an understanding of the distinct characteristics of those cells. Additional detailed information is provided on selected signal pathways in CNS tumors.

\title{
How to reference
}

In order to correctly reference this scholarly work, feel free to copy and paste the following:

Kumaravel Somasundaram, Soumya Alige Mahabala Rao and Zahid Nawaz (2011). MicroRNA (miRNA) Regulation in Glioma: Implications in Development, Progression, Grading, Prognosis and Therapy, Molecular Targets of CNS Tumors, Dr. Miklos Garami (Ed.), ISBN: 978-953-307-736-9, InTech, Available from: http://www.intechopen.com/books/molecular-targets-of-cns-tumors/microrna-mirna-regulation-in-gliomaimplications-in-development-progression-grading-prognosis-and-th

\section{INTECH}

open science | open minds

\author{
InTech Europe \\ University Campus STeP Ri \\ Slavka Krautzeka 83/A \\ 51000 Rijeka, Croatia \\ Phone: +385 (51) 770447 \\ Fax: +385 (51) 686166 \\ www.intechopen.com
}

\author{
InTech China \\ Unit 405, Office Block, Hotel Equatorial Shanghai \\ No.65, Yan An Road (West), Shanghai, 200040, China \\ 中国上海市延安西路65号上海国际贵都大饭店办公楼 405 单元 \\ Phone: +86-21-62489820 \\ Fax: $+86-21-62489821$
}


(C) 2011 The Author(s). Licensee IntechOpen. This chapter is distributed under the terms of the Creative Commons Attribution-NonCommercialShareAlike-3.0 License, which permits use, distribution and reproduction for non-commercial purposes, provided the original is properly cited and derivative works building on this content are distributed under the same license. 